\title{
Healthy Habits During the COVID-19 Pandemic
}

\section{Methods}

- SSRS Telephone Omnibus

- RDD landline and cell phones (40/60\% split)

- Conducted in English and Spanish

- General adult population, age $18+(100 \%$ incidence)

- October 22-November 9, 2020: $n=2,013$ adults 18 and over

$\circ$ (including $n=1,100$ adults 50 and over)

\section{Vendor}

AUS Marketing Research Systems, Inc. conducted this survey on behalf of AARP.

For further information, please visit their website: http://ausinc.com/ssrs/ 


\section{Questionnaire}

Q1. How would you rate your overall health? Would you say it is excellent, very good, good, fair, or poor?

\begin{tabular}{|l|c||c|c|}
\hline & $\begin{array}{c}\text { Total } \\
(\mathrm{n}=2,013)\end{array}$ & $\begin{array}{c}18-49 \\
(\mathrm{n}=1,078)\end{array}$ & $\begin{array}{c}50+ \\
(\mathrm{n}=934)\end{array}$ \\
\hline Excellent & $20 \%$ & $25 \% \%^{*}$ & $15 \%$ \\
\hline Very good & $32 \%$ & $33 \%$ & $31 \%$ \\
\hline Good & $32 \%$ & $31 \%$ & $33 \%$ \\
\hline Fair & $12 \%$ & $9 \%$ & $15 \%^{*}$ \\
\hline Poor & $3 \%$ & $1 \%$ & $4 \%^{*}$ \\
\hline
\end{tabular}

Q2. How would you describe your overall mental health at this point in time? Would you say it is excellent, very good, good, fair, or poor?

\begin{tabular}{|l|c||c|c|}
\hline & $\begin{array}{c}\text { Total } \\
(\mathrm{n}=2,013)\end{array}$ & $\begin{array}{c}18-49 \\
(\mathrm{n}=1,078)\end{array}$ & $\begin{array}{c}50+ \\
(\mathrm{n}=934)\end{array}$ \\
\hline Excellent & $30 \%$ & $31 \%$ & $29 \%$ \\
\hline Very good & $30 \%$ & $29 \%$ & $33 \%$ \\
\hline Good & $27 \%$ & $26 \%$ & $28 \%$ \\
\hline Fair & $10 \%$ & $11 \% \%^{*}$ & $8 \%$ \\
\hline Poor & $3 \%$ & $3 \%$ & $2 \%$ \\
\hline
\end{tabular}

Q3. Compared to before the coronavirus pandemic, do you now do more, less, or the same amount of the following activities?

\begin{tabular}{|l|c|c|c|c|}
\hline \multicolumn{1}{|c|}{ Total (n=2,013) } & More & $\begin{array}{c}\text { About the } \\
\text { Same }\end{array}$ & Less & $\begin{array}{c}\text { Never } \\
\text { did }\end{array}$ \\
\hline Drink alcoholic beverages & $8 \%$ & $47 \%$ & $19 \%$ & $25 \%$ \\
\hline Sleep or nap to make the time go by & $14 \%$ & $57 \%$ & $13 \%$ & $15 \%$ \\
\hline Smoke cigarettes & $5 \%$ & $25 \%$ & $11 \%$ & $58 \%$ \\
\hline $\begin{array}{l}\text { Take prescription medications in ways other } \\
\text { than prescribed }\end{array}$ & $2 \%$ & $47 \%$ & $7 \%$ & $49 \%$ \\
\hline $\begin{array}{l}\text { Eat unhealthy foods such as sweets, chips, } \\
\text { candy }\end{array}$ & $14 \%$ & $57 \%$ & $24 \%$ & $5 \%$ \\
\hline $\begin{array}{l}\text { Eat "comfort foods" meaning foods that } \\
\text { provides a nostalgic or sentimental value }\end{array}$ & $16 \%$ & $61 \%$ & $13 \%$ & $9 \%$ \\
\hline Exercise or walk vigorously & $23 \%$ & $52 \%$ & $21 \%$ & $3 \%$ \\
\hline Meditate or pray & $28 \%$ & $57 \%$ & $5 \%$ & $10 \%$ \\
\hline $\begin{array}{l}\text { Use CBD (Cannabidiol) oils or marijuana for } \\
\text { any reason, either recreational or medicinal }\end{array}$ & $5 \%$ & $23 \%$ & $7 \%$ & $65 \%$ \\
\hline $\begin{array}{l}\text { Take herbal products or dietary supplements } \\
\text { (such as black cohosh, echinacea, garlic, } \\
\text { ginkgo, saw palmetto, and St. John's wort) }\end{array}$ & $7 \%$ & $43 \%$ & $8 \%$ & $42 \%$ \\
\hline Overreact or lose control & $11 \%$ & $55 \%$ & $13 \%$ & $19 \%$ \\
\hline
\end{tabular}


Q4. On a scale of 1 to 10 , where 1 is extremely low and 10 is extremely high, please rate the overall level of stress in your life today?

\begin{tabular}{|l|c||c|c|}
\hline & $\begin{array}{c}\text { Total } \\
(n=2,013)\end{array}$ & $\begin{array}{c}18-49 \\
(n=1,078)\end{array}$ & $\begin{array}{c}50+ \\
(n=934)\end{array}$ \\
\hline Top 3 Box (8, 9, 10-extremely high stress) & $18 \%$ & $19 \%$ & $17 \%$ \\
\hline Middle 4 Box & $55 \%$ & $58 \%{ }^{*}$ & $52 \%$ \\
\hline Bottom 3 Box (1, 2, 3-extremely low stress) & $26 \%$ & $23 \%$ & $35 \%{ }^{*}$ \\
\hline
\end{tabular}

Q5. Have you, or do you plan to get a flu shot this year?

\begin{tabular}{|l|c||c|c|}
\hline & $\begin{array}{c}\text { Total } \\
(n=2,013)\end{array}$ & $\begin{array}{c}18-49 \\
(n=1,078)\end{array}$ & $\begin{array}{c}50+ \\
(n=934)\end{array}$ \\
\hline Yes, I have already gotten a flu shot & $41 \%$ & $32 \%$ & $52 \%{ }^{*}$ \\
\hline Not yet, but I plan to & $23 \%$ & $24 \%$ & $21 \%$ \\
\hline No, and I do not plan to & $35 \%$ & $43 \%{ }^{*}$ & $26 \%$ \\
\hline
\end{tabular}

Q6. What is your current employment status?

\begin{tabular}{|l|c||c|c|}
\hline & $\begin{array}{c}\text { Total } \\
(\mathrm{n}=2,013)\end{array}$ & $\begin{array}{c}18-49 \\
(\mathrm{n}=1,078)\end{array}$ & $\begin{array}{c}50+ \\
(\mathrm{n}=934)\end{array}$ \\
\hline Employed [NET] & $65 \%$ & $84 \%^{*}$ & $43 \%$ \\
\hline Employed, full-time & $53 \%$ & $69 \%^{*}$ & $34 \%$ \\
\hline Employed, part-time & $12 \%$ & $15 \%{ }^{*}$ & $9 \%$ \\
\hline Not Employed [NET] & & & \\
\hline Retired & $34 \%$ & $15 \%$ & $56 \%{ }^{*}$ \\
\hline Homemaker & $20 \%$ & ---- & $43 \%$ \\
\hline Student & $4 \%$ & $4 \%$ & $3 \%$ \\
\hline Temporarily unemployed & $2 \%$ & $4 \%$ & ---- \\
\hline Disabled or handicapped & $5 \%$ & $6 \%{ }^{*}$ & $4 \%$ \\
\hline
\end{tabular}

\section{Methodology}

SSRS conducted telephone interviews for AARP among a nationally representative sample of U.S. adults ages 18 and older. Interviews were conducted from October 22-November 9, 2020 among 2,013 adults age 18 or older, including 1,100 age 50 or older. Random Digit Dialing (RDD) was used for both landlines and cell phones. The data were weighted by age, race, sex, region, education, and marital status to reflect national population estimates. Overall results have a margin of sampling error of plus or minus 2.4 percentage points, including design effects due to weighting. Sampling, data collection, and tabulation by SSRS of Glen Mills, PA. More information about SSRS can be obtained by visiting www.ssrs.com. 